\title{
High-Performance Control of Dual-Inertia Servo-Drive Systems Using Low-Cost Integrated SAW Torque Transducers
}

\author{
Tim M. O'Sullivan, Chris M. Bingham, Member, IEEE, and Nigel Schofield
}

\begin{abstract}
This paper provides a systematic comparative study of compensation schemes for the coordinated motion control of two-inertia mechanical systems. Specifically, classical proportional-integral (PI), proportional-integral-derivative (PID), and resonance ratio control (RRC) are considered, with an enhanced structure based on RRC, termed RRC+, being proposed. Motor-side and load-side dynamics for each control structure are identified, with the "integral of time multiplied by absolute error" performance index being employed as a benchmark metric. PID and RRC control schemes are shown to be identical from a closed-loop perspective, albeit employing different feedback sensing mechanisms. A qualitative study of the practical effects of employing each methodology shows that RRC-type structures provide preferred solutions if low-cost high-performance torque transducers can be employed, for instance, those based on surface acoustic wave technologies. Moreover, the extra degree of freedom afforded by both PID and RRC, as compared with the basic PI, is shown to be sufficient to simultaneously induce optimal closed-loop performance and independent selection of virtual inertia ratio. Furthermore, the proposed $\mathrm{RRC}+$ scheme is subsequently shown to additionally facilitate independent assignment of closed-loop bandwidth. Summary attributes of the investigation are validated by both simulation studies and by realization of the methodologies for control of a custom-designed two-inertia system.
\end{abstract}

Index Terms-Acceleration control, motion control, resonance, surface acoustic wave (SAW) devices, torque control, velocity control, vibration control.

\section{NOMENCLATURE}

$J_{m} \quad$ Motor inertia $\left(\mathrm{kg} \cdot \mathrm{m}^{2}\right)$.

$J_{d} \quad$ Load inertia $\left(\mathrm{kg} \cdot \mathrm{m}^{2}\right)$.

$J_{t} \quad$ Total system inertia $\left(\mathrm{kg} \cdot \mathrm{m}^{2}\right)$.

$K_{\text {md }} \quad$ Interconnecting shaft stiffness $(\mathrm{N} \cdot \mathrm{m} / \mathrm{rad})$.

$R=J_{d} / J_{m}$. Inertia ratio.

$t_{e} \quad$ Motor electromagnetic torque $(\mathrm{N} \cdot \mathrm{m})$.

$t_{d} \quad$ Load-side torque $(\mathrm{N} \cdot \mathrm{m})$.

$t_{\mathrm{md}} \quad$ Torsional shaft torque $(\mathrm{N} \cdot \mathrm{m})$.

$\omega_{n} \quad$ Mechanical resonant frequency $(\mathrm{rad} / \mathrm{s})$.

Manuscript received August 17, 2004; revised October 20, 2004. Abstract published on the Internet May 18, 2006. This work was supported in part by the U.K. Engineering and Physical Sciences Research Council (EPSRC) and in part by Sensor Technology, Ltd., Banbury, U.K.

T. M. O'Sullivan and C. M. Bingham are with the Electrical Machines and Drives Group, Department of Electronic and Electrical Engineering, University of Sheffield, S1 3JD Sheffield, U.K. (e-mail: c.bingham@sheffield.ac.uk).

N. Schofield is with the Power Conversion Group, School of Electrical and Electronic Engineering, University of Manchester, M60 1QD Manchester, U.K.

Digital Object Identifier 10.1109/TIE.2006.878311

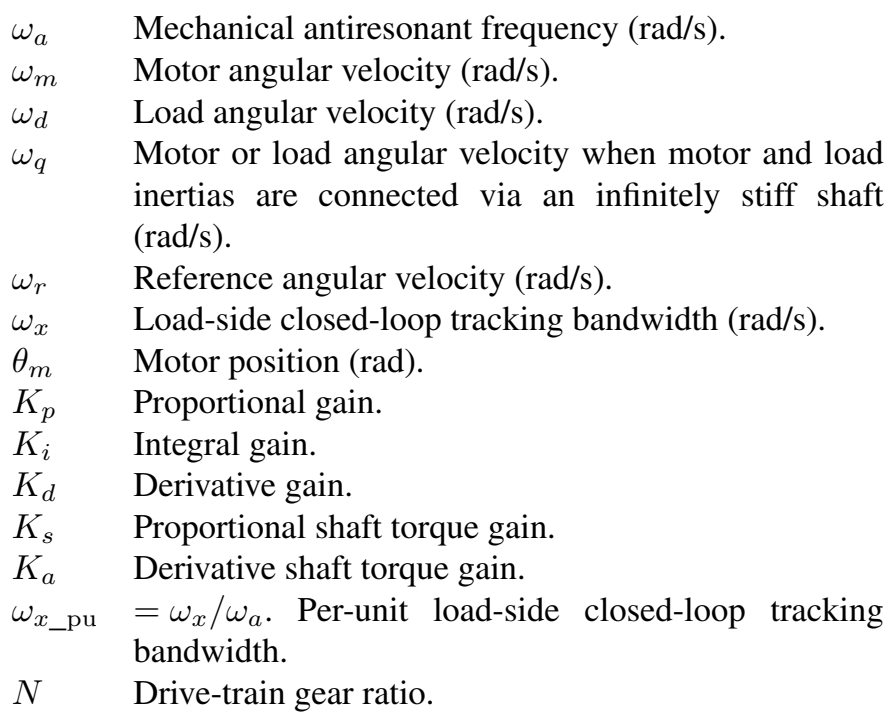

\section{INTRODUCTION}

$\mathbf{R}$ ECENT improvements in power density of electromagnetic machines and power conversion electronics have allowed electromagnetic systems to encroach into application fields that have traditionally been the domain of mechanical and hydraulic systems. In these cases, torque production can be considered essentially instantaneous, limited predominantly by the bandwidth limitations of feedback sensors, viz. phase current transducers, and position transducers such as resolvers and optical encoders, etc., with the system dynamics being dominated by the (often complex) mechanical drive train. Consequently, for complex multicomponent mechanical drive trains incorporating many nonstiff interconnecting shafts and elastic couplings, impulsive transient torque output from the servo drive can excite mechanical torsional resonances, ultimately leading to controller instability [1]. Moreover, the dominant fundamental resonant frequency for many such systems is typically $<300 \mathrm{~Hz}$, which often overlaps with the closedloop dynamic bandwidth imposed by the servo-drive control algorithm, with other higher resonant modes usually remaining relatively unexcited, thereby allowing a large proportion of servo-drive systems to be modeled using a two-inertia approximate model of the system mechanics [1], [2], [4]-[6], [9], [10].

While classical proportional-integral-derivative (PID) controllers are readily realizable and generally effective for a wide range of industrial control applications, the derivative 
contribution to the control action increases the sensitivity of the system to high-frequency unmodeled vibratory modes and measurement noise. Furthermore, an increasing number of motion control applications employ brushless ac machines with an integrated position sensor as the sole motion feedback sensor. If motor acceleration is required for feedback, it is obtained from the double derivative of sensed motor shaft position, which is usually from an incremental encoder employing a quadrature count. In practice, measurement noise ultimately bounds the high-frequency gain of the controller and, therefore, controller performance. Consequently, for high-bandwidth motion control applications, PID controllers cannot impart the desired levels of dynamic performance in the presence of extraneous inputs. A principle feature of this paper is therefore to demonstrate improved servo-drive performance for such systems by manipulating the closed-loop dynamics using constrained proprietary control architectures. Notably, therefore, this paper does not consider the use of more generic control design techniques, such as those based on $H_{\infty}$, for instance, since the flexibility required from the control architecture and the expertise to design such controllers are not commonly found for the general application of servo drives.

The advantages of incorporating measured shaft torque feedback have long been recognized as a solution for enhancing dynamic performance and imparting disturbance rejection properties in complex mechanical servo-drive systems. However, commonly employed torque transducers, such as those based on strain gauge and optical and inductive sensing device technologies, are often too mechanically compliant when incorporated in a drive train, further degrading stability margins and consequently requiring a reduction in closed-loop controller bandwidth to maintain transient performance, e.g., limited overshoot. Moreover, the additional capital cost associated with their integration has prohibited widespread application except in specialist areas where such expenditure does not constitute a primary design constraint, an example being a rolling mill application where the induced controller bandwidth is sufficiently low $(<10 \mathrm{~Hz})$ to enable the use of a capacitive shaft torque transducer to reduce torsional resonances [3]. Until recently, therefore, difficulties in acquiring reliable low-cost noninvasive shaft torque transducers have precluded their use in all but a minority of specialist closed-loop servo-drive systems, and recourse to observer-based state-estimation techniques has consequently been commonplace [4]-[6], [12]. In a simulation environment, state-variable controllers [13] are readily shown to provide enhanced control performance, with the observer bandwidth being assigned to be much higher than the system dynamics and all the states being assumed delay free and noise free. However, in practical high-performance servo-drive systems employing a single quantized feedback sensor, highbandwidth observers impart problematic noise-amplifying attributes (similar to the PID controller): stability problems arise primarily due to finite resolution of sensors and unmodeled resonant modes. Reducing observer bandwidth can attenuate sensor noise at the cost of deterioration in estimation accuracy and, ultimately, the controller performance. Consequently, observers are not commonly employed in servo-drive systems for the mass-market sector.

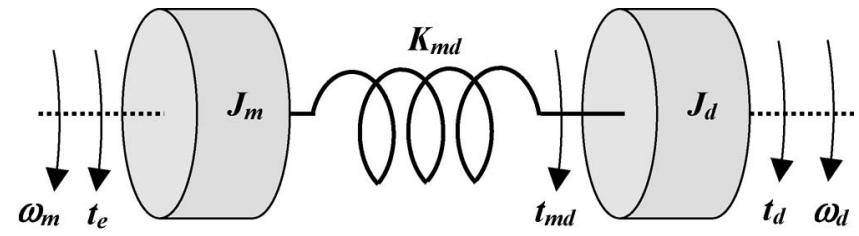

(a)

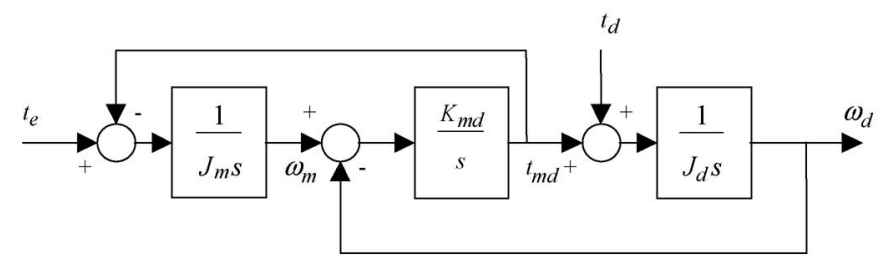

(b)

Fig. 1. Mechanical schematic. (a) Two-inertia representation of a servo-drive system. (b) Control block diagram.

Nevertheless, surface acoustic wave (SAW) torque sensors [7], [8] have recently emerged as a candidate technology for high-bandwidth applications employing electromechanical drive trains since they do not significantly affect system stiffness; exhibit high sensitivity, high signal-to-noise ratio, and high bandwidth; and are relatively unaffected by servomachine-generated electromagnetic noise and, hence, can be integrated directly within the servo-machine assembly. However, to realize the full dynamic potential offered by these devices, a reconsideration of classical control design techniques is required. In particular, while commercial servo-drive systems are tuned largely based on obtaining desirable performance with respect to motor shaft velocity, here, the performance of the drive train with respect to load-side dynamics is considered since this is ultimately what most applications require, and, notably, designing for the former can exhibit poor or undesirable load-side performance.

\section{Two-Inertia Mechanical System}

Fig. 1(a) provides a schematic of a two-inertia representation of a servo-drive system, where the motor angular velocity is denoted as $\omega_{m}$ and the load velocity is denoted as $\omega_{d}$. Since damping losses are usually considered to be relatively low, they are neglected without significantly affecting the accuracy of the forgoing analysis [1], [2], [4]-[6], [9], [10] [12], [13]. Fig. 1(b) provides a dynamic block diagram representation of the twoinertia mechanical system from which transfer functions describing the relationships between the electromagnetic torque produced by the servo machine, and the machine rotor angular velocity (1) and load angular velocity (2), can be derived, such as

$$
\begin{aligned}
\frac{\omega_{m}(s)}{t_{e}(s)} & =\frac{s^{2}+\omega_{a}^{2}}{J_{m} s^{3}+J_{m} \omega_{n}^{2} s} \\
\frac{\omega_{d}(s)}{t_{e}(s)} & =\frac{\omega_{a}^{2}}{J_{m} s^{3}+J_{m} \omega_{n}^{2} s}
\end{aligned}
$$




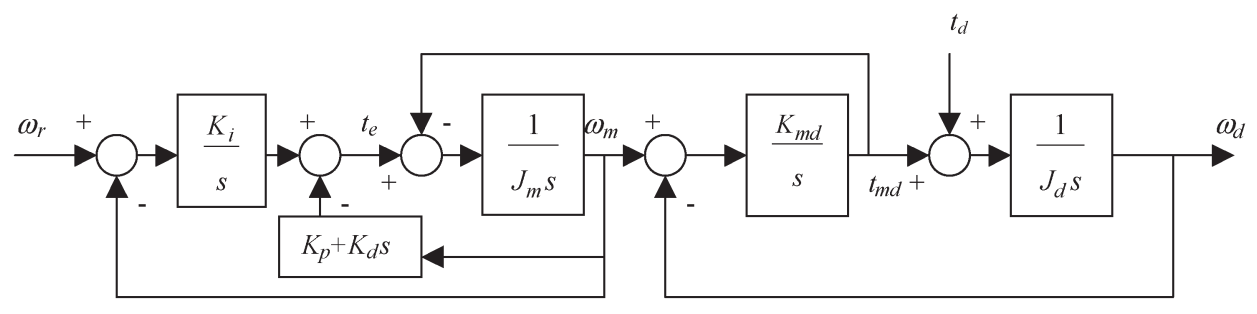

(a)

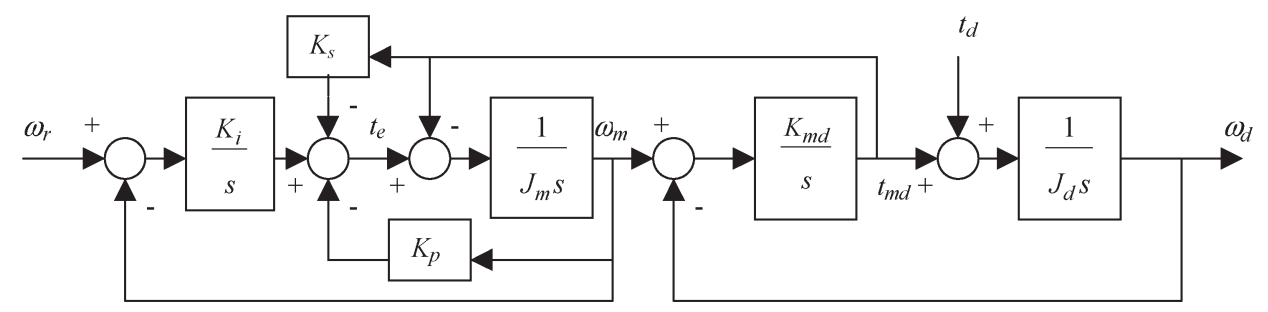

(b)

Fig. 2. Control block diagrams of the extended PI controllers for the two-inertia mechanical model. (a) PID controller. (b) RRC controller.

where $\omega_{a}$ (i.e., the antiresonant frequency) and $\omega_{n}$ (i.e., the resonant frequency) are defined as

$$
\begin{aligned}
& \omega_{a}=\sqrt{K_{\mathrm{md}}\left(\frac{1}{J_{d}}\right)} \\
& \omega_{n}=\omega_{a} \sqrt{R+1}
\end{aligned}
$$

and the load-to-motor inertia ratio is

$$
R=\frac{J_{d}}{J_{m}}
$$

From (4), the relationship between $\omega_{n}$ and $\omega_{a}$ is dependent on the load-to-motor inertia ratio $R$. Ultimately, $R$ will be shown to be crucial in determining the complexity of a controller to impart good closed-loop tracking performance and disturbance rejection properties.

\section{Proportional-Integral (PI), PID, And RESONANCE RATIO CONTROL (RRC) CONTROL STRUCTURES}

The forgoing analysis assumes that a sensor for feedback of motor velocity is present on the motor shaft (or derived from a suitable position measurement) since, notably, it has been previously demonstrated [2] that no fundamental performance benefit is gained by measuring load velocity (as opposed to motor velocity) for closed-loop control (of either the motor or load) in a two-inertia system, and the presented situation is the more usual in a commercial turnkey system.

Fig. 2(a) shows the structure of a classical PI control scheme with an additional derivative signal proportional to motor acceleration being included to form a PID structure, where $K_{d}$ is the derivative feedback gain. An alternative structure is given in Fig. 2(b), which takes the PI elements of Fig. 2(a) and augments them with a feedback signal proportional to shaft torque, where the associated feedback gain is $K_{s}$. The use of a feedback signal proportional to torsional torque in this manner has been commonly referred to as RRC [4]-[6]. Theoretically, it can be shown that the PID and RRC controllers are able to induce identical closed-loop dynamics, with the practical distinction between the two being dependent on the capability of the associated feedback sensor and processing interface - an important aspect that will be considered further in Section V-C.

Notably, the dynamics of the servo-amplifier current controller, which is governed primarily by delay time and $-3-\mathrm{dB}$ bandwidth, are assumed to be sufficient to have negligible effect on the system response, thereby allowing the servo amplifier to be modeled by a unity gain. A key limiting factor for raising the dynamic bandwidth of the two-inertia system is ultimately the response of the speed loop, which is constrained by mechanical resonances and the attributes of the feedback sensor, by the effects of noise injection (since speed is generally obtained from the derivative of quantized position) and sensor resonance induced by the physical mounting of the sensor to the motor (not specifically considered herein) [14].

\section{A. PI, PID, and RRC Tracking Dynamics}

From the PI control structure of Fig. 2(a), the closed-loop transfer functions from reference velocity $\omega_{r}$ to motor velocity $\omega_{m}$, and from reference velocity to load velocity $\omega_{d}$ (tracking 
dynamics), can be derived, resulting in (6) and (7), respectively, where $\tilde{J}_{m}=J_{m}$ and $\tilde{R}=R$, viz.

$$
\begin{aligned}
& \frac{\omega_{m}(s)}{\omega_{r}(s)} \\
& =\frac{K_{i}\left(s^{2}+\omega_{a}^{2}\right)}{\tilde{J}_{m} s^{4}+K_{p} s^{3}+\left(\tilde{J}_{m} \omega_{a}^{2}(1+\tilde{R})+K_{i}\right) s^{2}+K_{p} \omega_{a}^{2} s+K_{i} \omega_{a}^{2}} \\
& =\frac{K_{i}\left(s^{2}+\omega_{a}^{2}\right)}{\tilde{J}_{m} s^{2}\left(s^{2}+\omega_{a}^{2}(1+\tilde{R})\right)+\left(K_{p} s+K_{i}\right)\left(s^{2}+\omega_{a}^{2}\right)} \\
& \frac{\omega_{d}(s)}{\omega_{r}(s)} \\
& =\frac{K_{i} \omega_{a}^{2}}{\tilde{J}_{m} s^{4}+K_{p} s^{3}+\left(\tilde{J}_{m} \omega_{a}^{2}(1+\tilde{R})+K_{i}\right) s^{2}+K_{p} \omega_{a}^{2} s+K_{i} \omega_{a}^{2}} \\
& =\frac{K_{i} \omega_{a}^{2}}{\tilde{J}_{m} s^{2}\left(s^{2}+\omega_{a}^{2}(1+\tilde{R})\right)+\left(K_{p} s+K_{i}\right)\left(s^{2}+\omega_{a}^{2}\right)} .
\end{aligned}
$$

By observation, if the closed-loop bandwidth $\omega_{x} \ll \omega_{a}$ (i.e., $\left(s^{2}+\omega_{a}^{2}\right) \approx \omega_{a}^{2}$ and $\left.\left(s^{2}+\omega_{a}^{2}(1+\tilde{R})\right) \approx \omega_{a}^{2}(1+\tilde{R})\right)$, then $(6)$ and (7) reduce to the second-order lumped-inertia approximation (8), as is commonly used for autotuning of servo-drive systems, i.e.,

$$
\frac{\omega_{q}(s)}{\omega_{r}(s)}=\frac{K_{i}}{\tilde{J}_{m}(1+\tilde{R}) s^{2}+K_{p} s+K_{i}} .
$$

Here, there is no distinction between the motor-side and loadside dynamics since it is assumed that the inertias are connected via an infinitely stiff shaft, and $\omega_{q}$ therefore represents either $\omega_{m}$ or $\omega_{d}$. More generally, the closed-loop tracking dynamics for the PID and RRC controllers are similar except that the denominators of the transfer functions contain "virtual" mechanical parameters. Specifically, for the PID case, the equivalent motor inertia $\tilde{J}_{m}$ and the virtual inertia ratio $\tilde{R}$ are

$$
\tilde{J}_{m}=J_{m}+K_{d} \quad \tilde{R}=\frac{J_{d}}{\tilde{J}_{m}}
$$

whereas for the RRC case, $\tilde{J}_{m}$ and $\tilde{R}$ are

$$
\tilde{J}_{m}=J_{m} \quad \tilde{R}=R\left(1+K_{S}\right) \quad(\mathrm{RRC}) .
$$

By inspection of (6) and (7), it can be seen that feedback measurements of either torsional torque or motor acceleration (i.e., the derivative of angular velocity) result in identical controllerinduced dynamics, both enabling a change in the virtual inertia ratio $\tilde{R}$.

\section{B. Performance Criteria}

Controller-induced closed-loop performance imparted by the PID and RRC schemes and an enhanced scheme proposed in
TABLE I

MeChANICAL PARAMETERS

\begin{tabular}{l|c}
\hline$R$ & $0.5,1,2$ and \\
& 50 (simulation only) \\
$J_{d}$ & $0.0027 \mathrm{kgm}^{2}$ \\
$K_{m d}$ & $125 \mathrm{Nm} / \mathrm{rad}$ \\
$\omega_{a}$ & $215.16 \mathrm{rad} / \mathrm{s} \quad(34 \mathrm{~Hz})$ \\
\hline
\end{tabular}

Section IV are evaluated using the integral of time multiplied by absolute error (ITAE) criterion for a step reference input. The ITAE performance index provides the best performance selectivity by minimizing overshoot and settling time for a given rise time or equivalent $-3-\mathrm{dB}$ bandwidth [11]. Closedloop load-side tracking behaviors given by $\omega_{d}(s) / \omega_{r}(s)$, which are imparted by the PID and RRC control schemes, are completely defined by their pole locations (since there are no closed-loop zeros), thereby enabling the required characteristic equations for each to be specified by the respective optimal ITAE indexes. Specifically, for the second-order and fourthorder characteristic equations, the optimal ITAE polynomials are given by (11) and (12), respectively, where $\omega_{x}$ represents the $-3-\mathrm{dB}$ bandwidth, i.e.,

$$
\begin{aligned}
& s^{2}+1.4 \omega_{x} s+\omega_{x}^{2} \\
& s^{4}+2.1 \omega_{x} s^{3}+3.4 \omega_{x}^{2} s^{2}+2.7 \omega_{x}^{3} s+\omega_{x}^{4} .
\end{aligned}
$$

\section{Simulation of a Two-Inertia Test Facility}

An experimental two-inertia test facility comprising $2 \times$ $2.2 \mathrm{~kW}$ (Control Techniques, Ltd., Unimotor) brushless permanent-magnet servo machines, exited by servo amplifiers (Control Techniques, Ltd., Unidrive SP), interconnected via an inline SAW-based instrumental torque transducer, has been assembled. Mechanical parameters of the test facility are given in Table I for chosen values of $R$, which are achieved by inserting disks of various inertia to the motor-side servo machine. Using the presented control structures, dynamic simulation models of the experimental closed-loop system are now used to provide performance comparisons.

Initially, PI controller gains $K_{p}$ and $K_{i}$ are chosen to assign the optimal second-order ITAE characteristic equation (11) to the simplified lumped-inertia model given in (8) and the fourthorder system represented by (6) and (7). In this case, the fourthorder model, which is tuned with the optimal second-order parameters, is compared with the optimal second-order step response. For example, Fig. 3 shows the difference in the ITAE of the load-side step responses of the fourth-order (optimal) and second-order models. More generally, step responses of the fourth-order system can be compared with the respective optimal second-order step responses, as the per-unit load-side closed-loop tracking bandwidth $\omega_{x \_ \text {pu }}=\omega_{x} / \omega_{a}$ and $R$ are varied. Results of the investigation are given in Fig. 4, and identifying the frequency range over which the single lumpedinertia approximation provides an acceptable model of the two-inertia mechanical system. In particular, Fig. 4(a) shows the ITAE of the difference between the step responses at the motor side, whereas Fig. 4(b) shows the ITAE of the difference 


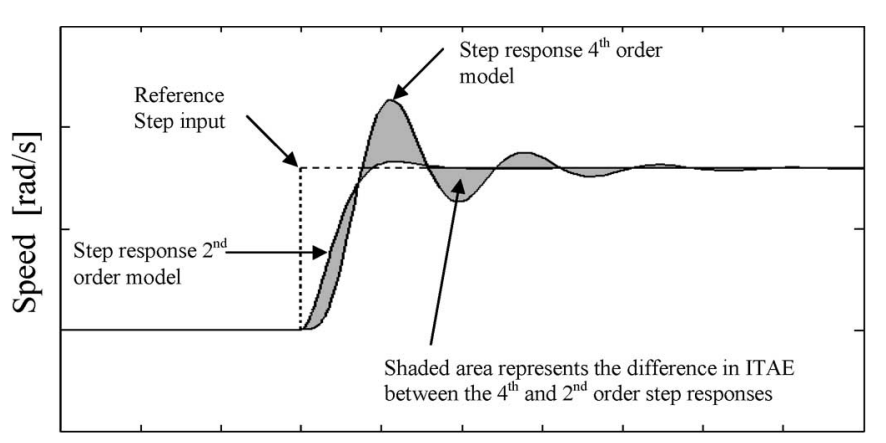

Time [s]

Fig. 3. Example of the difference in the ITAE calculation of the load-side step responses for the fourth-order and second-order (optimal) models.

between the step responses at the load side. The following trends can be identified.

1) When $R=1$ and $\omega_{x \_ \text {pu }}<0.4$, the single lumped-inertia simplification is seen to provide a good estimation of drive-train performance and is largely insensitive to increases in $R$. For instance, Fig. 5 shows tracking responses (step demand of $10 \mathrm{rad} / \mathrm{s}$ ) for the motor side (6) and the load side (7), with the controller optimally tuned for (8), for cases when $\omega_{x \_ \text {pu }}=0.4$ and $\omega_{x \_ \text {pu }}=0.88$ and $R=1$ for both cases.

2) When $R<1$, performance is seen to significantly degrade, and hence, the tuning mechanism based on the second-order approximation is not appropriate in this region. This feature is particularly noticeable in the loadside performance [Fig. 4(b)].

It is concluded therefore that tuning a two-inertia system according to motor-side performance objectives, i.e., using sensors mounted directly on the motor shaft, or by assuming a lumped-inertia approximation to select the velocity controller parameters, as is commonplace in commercial drive-system tuning algorithms, may result in undesirable load-side performance, particularly when the load inertia is smaller than the motor inertia. Importantly, these results are also generic to both the PID and RRC structures, with $R$ replaced by $\tilde{R}$, albeit implemented with different feedback sensors, viz. derivative of angular velocity for the former and shaft torque feedback for the latter, since the additional complexity provided by these schemes only allows independent control of $\tilde{R}$.

From trend 1 , when $\omega_{x \_ \text {pu }}>0.4$, tuning the two-inertia mechanical system according to the fourth-order models (6) and (7) and the fourth-order optimal ITAE index (12) should be considered. Equating coefficients of the denominator of (7) and (12) then provides expressions for the optimal inertia ratio and solutions for the optimal per-unit load-side closedloop tracking bandwidth ratio $\omega_{x \_p u}$. Specifically, equating coefficients requires $R=1.08$ and $\omega_{x \_ \text {pu }}=0.88$ for optimality. By analyzing closed-loop pole restrictions [2], the load-side tracking performance imparted by the PI controller for the fourth-order model shows increasingly underdamped tendencies as $R$ reduces from the optimal value or as $\omega_{x}>\omega_{a}$. However, the requirement for $R \approx 1$ can be problematic for many servo-drive systems, particularly when a gear-reduction stage is employed between the motor and load, which reduces the effective load inertia (from the perspective of the motor) by the square of the gear ratio $N^{2}$. Although insufficient degrees of freedom are available in the PI controller to independently assign the optimal value of $R$, sufficient freedom does exist using the PID and RRC control structures. However, there remains no flexibility to independently select the optimal loadside closed-loop tracking bandwidth ratio; that is, to satisfy the optimal ITAE criteria, it still has to be $\omega_{x \_p u}=0.88$. Moreover, in practice, the maximum available "control energy" and the quality of the feedback sensor signal also place practical limits on the virtual inertia ratio. Table II summarizes the optimal (fourth-order ITAE polynomial) PI, PID, and RRC controller gains as a function of the mechanical parameters.

By changing the polarity of the additional feedback gains $K_{s}$ and $K_{d}, \tilde{R}$ can be virtually reduced, if desired, to obtain optimum load-side tracking performance when $R>1$. However, as previously shown in Fig. 4, such a reduction does not result in significant performance enhancement. For example, the PI controller is tuned according to the equations given in Table II (with $K_{d}=0$ ) as $R$ is increased ( $R \geq 1$ ) by adjusting $J_{m}$. For each new value of inertia ratio, $K_{p}$ and $K_{i}$ are recalculated assuming $J_{d}=J_{t} / 2$, where $J_{t}$ is the total system inertia, i.e., $J_{t}=J_{m}(1+R)$, with the resulting step responses given in Fig. 6 for various $R$. In this case, the additional complexity of the PID or RRC controllers is not necessary to virtually reduce $R$ since good performance is obtained from the basic PI scheme with $R \geq 1$. This has also been reported in [2].

\section{Regulation Dynamics}

Closed-loop transfer functions that relate the disturbance torque to load speed (regulation dynamics) have been derived in (13) and (14), respectively, shown at the bottom of the page, for the PI, PID, and RRC controllers of Fig. 2. The equivalent motor inertia $\widetilde{J}_{m}$ and the inertia ratio $\tilde{R}$ for the PID controller are given in (9) (note that $\tilde{J}_{m}=J_{m}$ and $\tilde{R}=R$ for PI), whereas the equivalent inertia ratio $\tilde{R}$ for the $\mathrm{RRC}$ controller is given in (10). Since the closed-loop regulation dynamics contain complex zeros, optimum regulation performance is not solely determined by pole locations. It is notable, however, that when the PID and RRC controllers for the two-inertia system are tuned for optimum tracking performance according

$$
\begin{aligned}
\frac{\omega_{d}}{t_{d}} & =\frac{\left(\tilde{J}_{m} s^{3}+K_{p} s^{2}+\left(K_{i}+K_{\mathrm{md}}\right) s\right) / J_{d}}{\tilde{J}_{m} s^{4}+K_{p} s^{3}+\left(\tilde{J}_{m} \omega_{a}^{2}(1+\tilde{R})+K_{i}\right) s^{2}+K_{p} \omega_{a}^{2} s+K_{i} \omega_{a}^{2}} \\
\frac{\omega_{d}}{t_{d}} & =\frac{\left(J_{m} s^{3}+K_{p} s^{2}+\left(K_{i}+K_{\mathrm{md}}\left(1+K_{s}\right)\right) s\right) / J_{d}}{J_{m} s^{4}+K_{p} s^{3}+\left(J_{m} \omega_{a}^{2}(1+\tilde{R})+K_{i}\right) s^{2}+K_{p} \omega_{a}^{2} s+K_{i} \omega_{a}^{2}}
\end{aligned}
$$




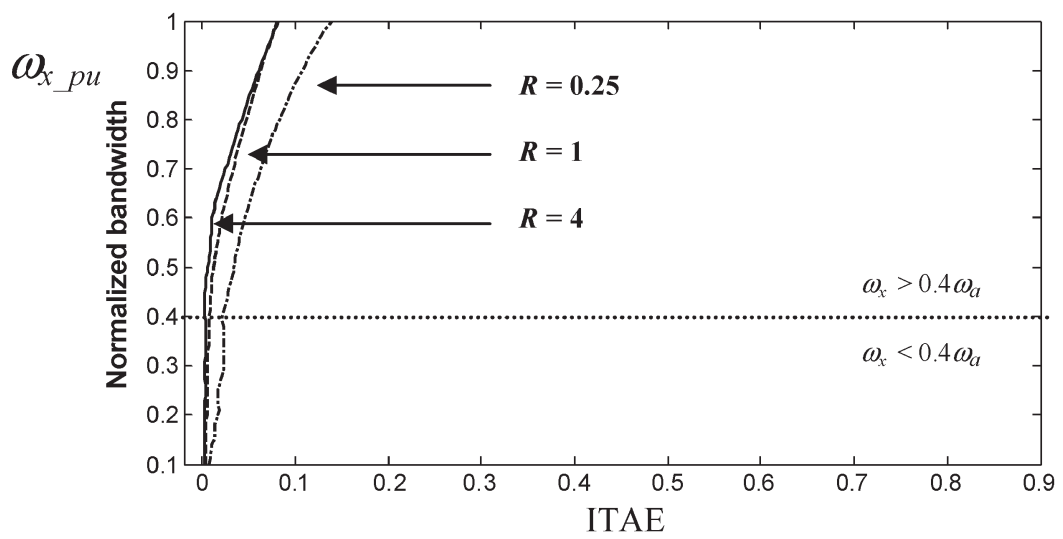

(a)

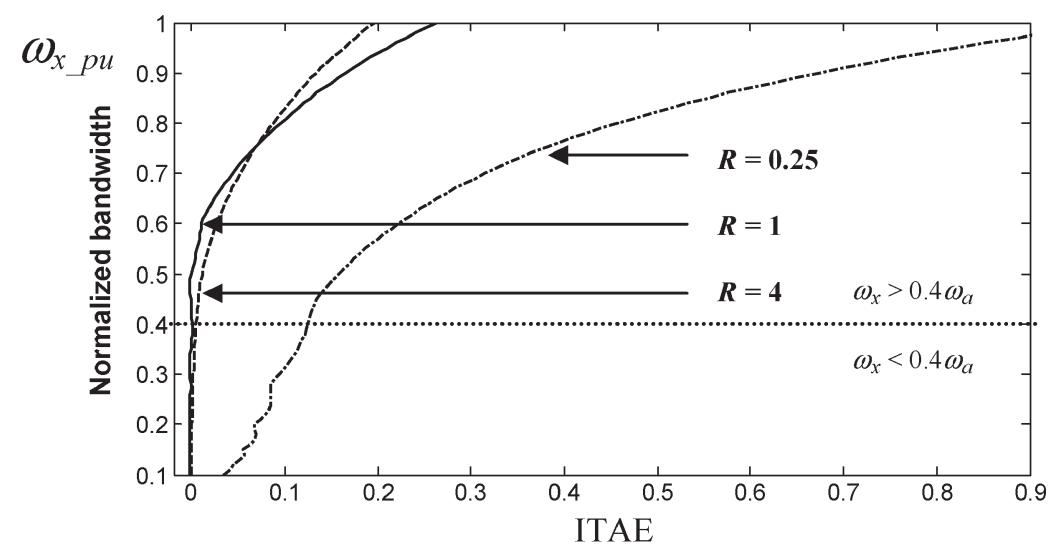

(b)

Fig. 4. ITAE difference calculation of the load-side step responses for the fourth-order and second-order (optimal) models. (a) Motor side. (b) Load side.

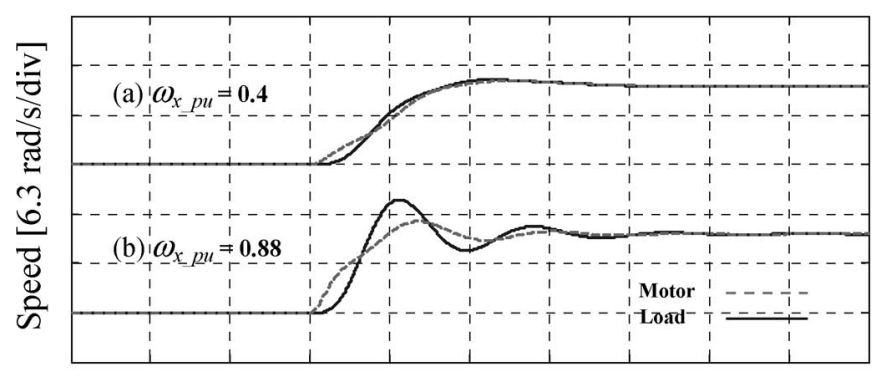

Time $[20 \mathrm{~ms} / \mathrm{div}]$

Fig. 5. Simulated tracking step response for the PI controller tuned to a second-order single lumped-inertia simplification, where $R=1$.

to the fourth-order model, the assignment of closed-loop zeros is identical. Hence, the PID and RRC controllers theoretically result in identical disturbance rejection properties.

To show the influence of $R$ on disturbance rejection performance, the method presented to generate the results of Fig. 6 for the tracking step responses is now repeated to obtain regulation response characteristics for a step load input of $2 \mathrm{~N} \cdot \mathrm{m}$; the results are given in Fig. 7. It is evident from Fig. 7 that similar performance characteristics are obtained as $R$ is varied.

\section{RRC+ CONTROL STRUCTURE}

To address inadequacies of the PID and RRC controllers to independently assign $\tilde{R}$ and the optimal per-unit load-side
TABLE II

PI, PID, AND RRC CONTROLLER GAINS FOR OPTIMAL LOAD-SIDE Tracking Performance

\begin{tabular}{c|c}
\hline PI, PID & RRC \\
\hline$K_{p} \approx 1.85 \omega_{a} J_{d}$ & $K_{p} \approx 1.85 \omega_{a} J_{m}$ \\
$K_{i} \approx 0.6 \omega_{a}{ }^{2} J_{d}$ & $K_{i} \approx 0.6 \omega_{a}{ }^{2} J_{m}$ \\
$K_{d}=J_{d}-J_{m}$ & $K_{s}=\frac{J_{m}}{J_{d}}-1$ \\
\hline
\end{tabular}

tracking bandwidth (fixed at $\omega_{x \_ \text {pu }}=0.88$ due to pole assignment restrictions), an extended control structure is now proposed (Fig. 8). Termed RRC+, the technique essentially constitutes state-variable feedback by employing the derivative of shaft torque, which is a variable not normally available without the addition of an extra speed sensor on the load side, or a state observer, but is now feasible due to recent developments in integrated SAW torque transducers. Notably, the derivative of shaft torque in a two-inertia system is given by

$$
\frac{d t_{\mathrm{md}}}{d t}=K_{\mathrm{md}}\left(\omega_{m}-\omega_{d}\right)
$$

i.e., the resulting feedback signal is, in essence, proportional to the difference in motor and load speeds. The resulting fourthorder RRC+ load-side tracking and regulation dynamics are 


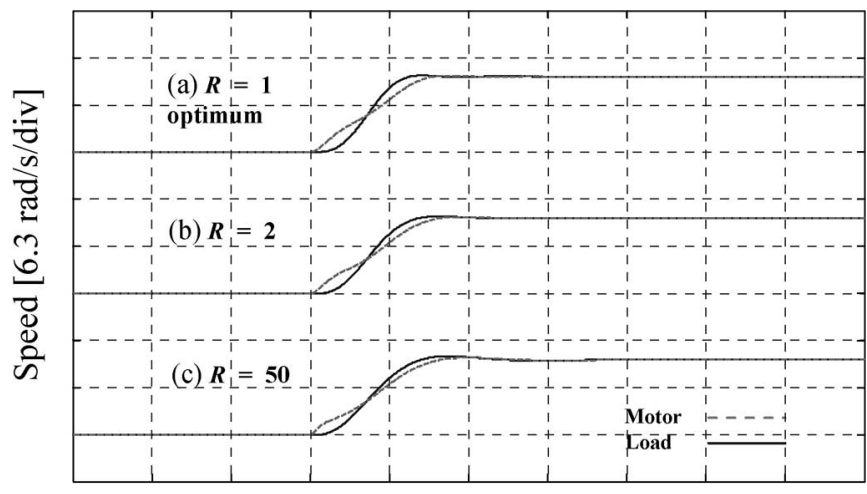

Time $[20 \mathrm{~ms} / \mathrm{div}]$

Fig. 6. Simulated tracking step response for the PI controller tuned to a twoinertia fourth-order model.

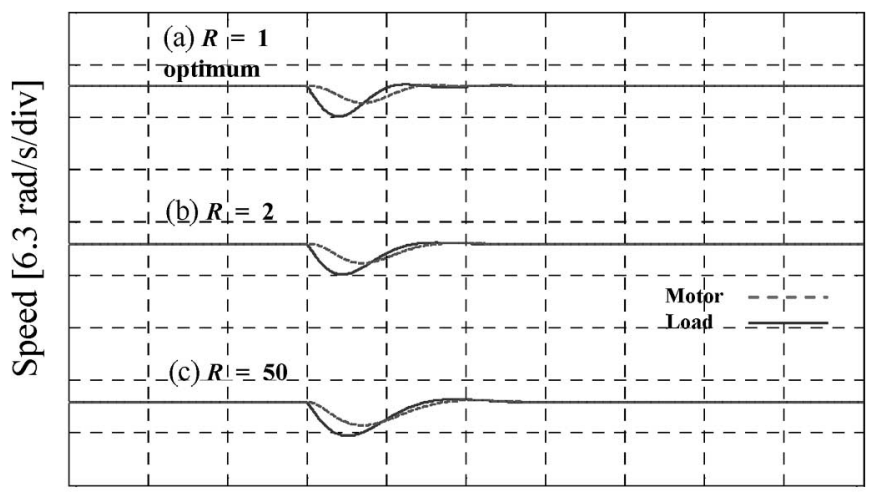

Time $[20 \mathrm{~ms} / \mathrm{div}]$

Fig. 7. Simulated regulation step response for the PI controller tuned to a twoinertia fourth-order model.

expressed in transfer function form by (16) and (17), respectively, shown at the bottom of the page.

Since four independently assignable gain variables are now present, namely 1) $K_{p}$,2) $K_{i}$,3) $K_{s}$, and 4) $K_{a}$, no theoretical restriction exists on the assignment of desired closed-loop poles, allowing the optimal fourth-order ITAE denominator polynomial (14) to be completely specified. To illustrate resulting time-domain and frequency-domain attributes, Fig. 9(a) shows the optimal closed-loop pole locations, whereas Fig. 9(b) shows tracking time-domain performance characteristics as $\omega_{x \_ \text {pu }}$ is varied. Table III gives the required controller gains for optimal load-side tracking performance. It is noteworthy that setting $\omega_{x \_ \text {pu }}=0.88$ results in $K_{a}=0$, thereby reducing the controller structure to that of RRC, and substituting $\omega_{x \_ \text {pu }}=$ 0.88 and $R=1$ into Table III results in $K_{d}=K_{s}=0$, further reducing the controller to that of PI.
For completeness, the effect of a change in load inertia (a common industrial occurrence) on the transient response (when $\omega_{x \_ \text {pu }}=1.4$ ) is shown in Fig. 9(c). Generally, as load inertia increases from the nominal value, overshoot and rise time increase. In contrast, for a reduction in inertia, rise time reduces, whereas overshoot remains relatively constant. In either case, however, a general reduction in performance ensues, as evidenced by the increase in ITAE. Moreover, for a reduction in the load inertia, the response becomes increasingly oscillatory by virtue of a reduction in the inertia ratio $R$.

Fig. 10 shows the dynamic tracking and regulation responses of the proposed RRC+ controller when $R=1$. The simulation begins with a $10-\mathrm{rad} / \mathrm{s}$ step demand followed by a $2 \mathrm{~N} \cdot \mathrm{m}$ step disturbance torque after $0.8 \mathrm{~s}$. In particular, Fig. 10(a) shows the motor-side and load-side step responses for $\omega_{x \_ \text {pu }}=1.4$, whereas Fig. 10(b) shows a comparison of load-side step responses of the PI controller (optimally tuned to $\omega_{x_{-} \mathrm{pu}}=0.88$ for $R=1)$ and RRC+ controller $\left(\omega_{x \_p u}=1.4\right)$. It is evident that increasing the optimal tracking bandwidth using the RRC+ controller decreases the overall transient response time as compared with the PI controller and improves both the tracking and regulation performance. For completeness, Table IV summarizes the optimal tracking and regulation performance criteria for the PI, PID, RRC, and RRC+ control schemes.

\section{EXPERIMENTAL VALIDATION}

\section{A. Experimental Apparatus and SAW Torque Transducer}

The control characteristics, simulation results, and presented observations are now validated on the experimental test facility described in Section III-C (see Fig. 11). Algorithms, sensor inputs, and controlled outputs are realized via a digital signal processor (DSP)-based hardware development platform, as illustrated in Fig. 11(b). Notably, the sampling rate used for all control schemes is $12 \mathrm{kHz}$, and all feedback signals are filtered using a 1-kHz bandwidth low-pass filter to reduce the effects of aliasing.

The $20 \mathrm{~N} \cdot \mathrm{m}$ SAW-based torque transducer contains two SAW devices mounted on a shaft of known stiffness and is used to realize RRC and RRC+. Each device consists of an array of thin-metal electrodes deposited at fractional wavelengths apart from a polished piezoelectric substrate. An RF signal applied to the electrodes excites a SAW over the device that resonates at a frequency determined by the distance between the interdigital metal electrodes. Torsion applied to the transducer creates two principle components of strain, namely: 1) $S_{x x}$ and 2) $S_{y y}$, subjecting one SAW device to tension and the other to compression, as illustrated schematically in Fig. 11(c). Strain varies the resonant frequency of the SAW devices, the outputs

$$
\begin{aligned}
& \frac{\omega_{d}}{\omega_{r}}=\frac{K_{i} \omega_{a}^{2}}{J_{m} s^{4}+\left(K_{p}+K_{a} K_{\mathrm{md}}\right) s^{3}+J_{m} \omega_{a}^{2}(1+\tilde{R})+K_{i} s^{2}+K_{p} \omega_{a}^{2} s+K_{i} \omega_{a}^{2}} \\
& \frac{\omega_{d}}{t_{d}}=\frac{\left(J_{m} s^{3}+\left(K_{p}+K_{a} K_{\mathrm{md}}\right) s^{2}+\left(K_{i}+K_{\mathrm{md}}\left(1+K_{s}\right)\right) s\right) / J_{d}}{J_{m} s^{4}+\left(K_{p}+K_{a} K_{\mathrm{md}}\right) s^{3}+J_{m} \omega_{a}^{2}(1+\tilde{R})+K_{i} s^{2}+K_{p} \omega_{a}^{2} s+K_{i} \omega_{a}^{2}}
\end{aligned}
$$




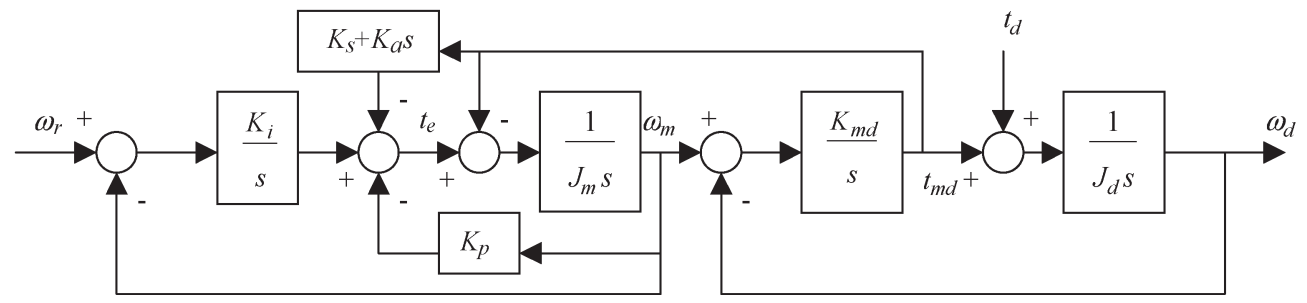

Fig. 8. RRC+ controller for a two-inertia mechanical model.

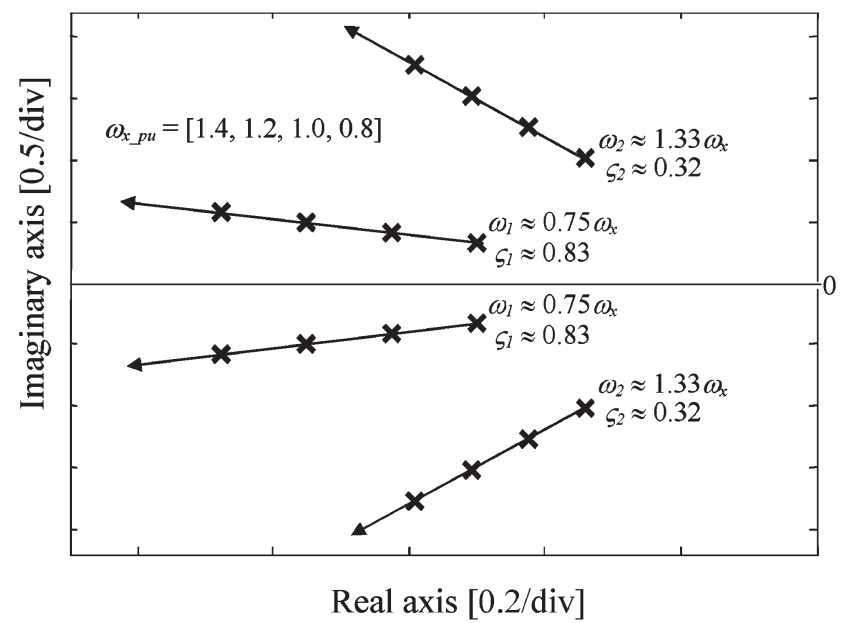

(a)

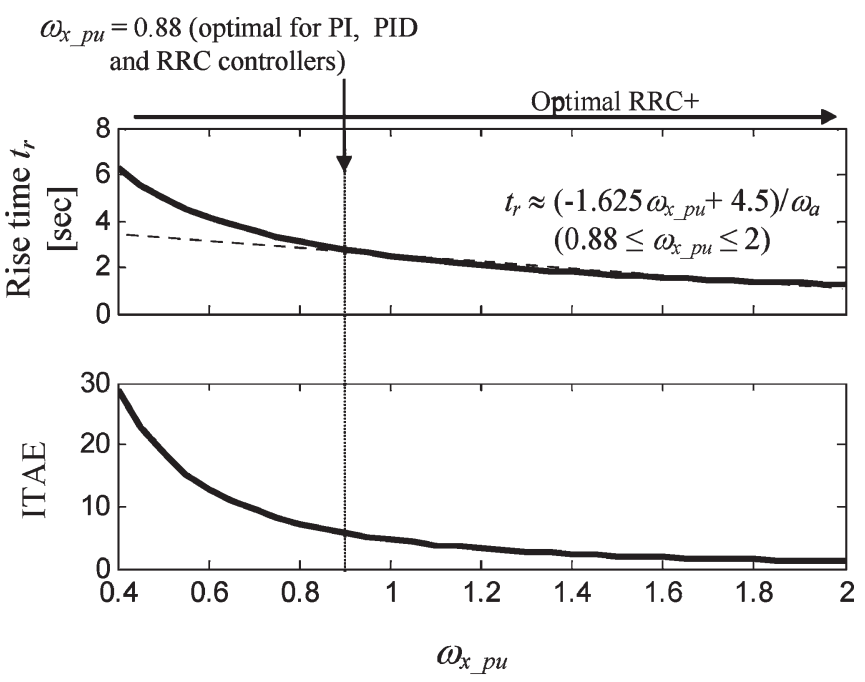

(b)

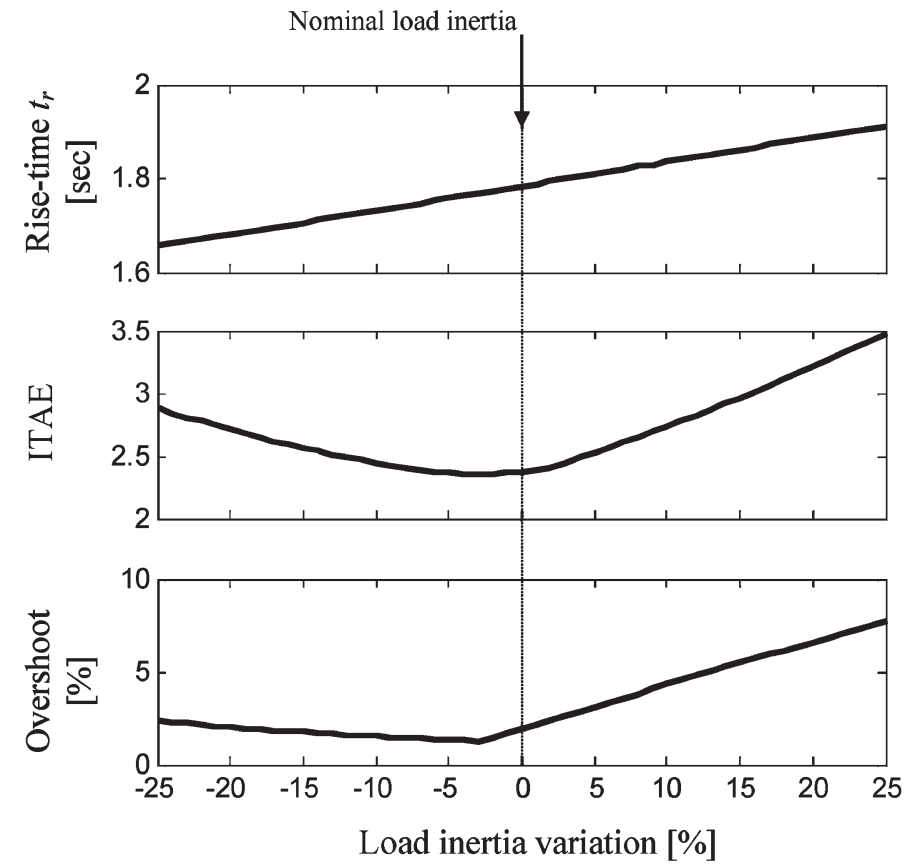

(c)

Fig. 9. Closed-loop load-side tracking performance characteristics, where $\omega_{a}=1 \mathrm{rad} / \mathrm{s}$. (a) Optimal ITAE pole locations. (b) Optimal ITAE step response as $\omega_{x \_ \text {pu }}$ is varied. (c) Effect of varying load inertia on the optimal ITAE step response, where nominally $R=1$ and $\omega_{x \_p u}=1.4$.

of which are connected to an RF coupler. After mixing and signal processing, the sum and difference frequencies provide shaft torque, temperature, and axial stress compensation outputs. Typically, the SAW resonant frequency is $500 \mathrm{MHz}$, with a $500-\mathrm{kHz}$ deviation equating to maximum strain [8]. This is a key advantage of SAW sensor technologies since it provides a simple noncontact coupling between the rotational devices and stationary processing unit, and, by careful design, the influence of electromagnetic interference can be minimized [7], [8]. Moreover, the temperature coefficient of the SAW devices 
TABLE III

RRC+ CONTROLLER GAINS FOR OPTIMAL LOAD-SIDE Tracking PERformance

\begin{tabular}{l|c}
\hline$K_{p}=2.7 \omega_{a} \omega_{x_{-} p u}{ }^{3} J_{m}$ & $K_{s}=\left(\frac{3.4 \omega_{x_{-} p u}{ }^{2}-\omega_{x_{-} p u}{ }^{4}-(1+R)}{R}\right)$ \\
$K_{i}=\omega_{a}{ }^{2} \omega_{x_{-} p u}{ }^{4} J_{m}$ & $K_{a}=\frac{\omega_{a} J_{m}}{K_{m d}}\left(2.1 \omega_{x_{-} p u}-2.7 \omega_{x_{-} p u}{ }^{3}\right)$ \\
\hline
\end{tabular}

is $<0.01 \%$ per ${ }^{\circ} \mathrm{C}$ for $-10{ }^{\circ} \mathrm{C}$ to $50{ }^{\circ} \mathrm{C}$ and $<0.15 \%$ per ${ }^{\circ} \mathrm{C}$ for $40{ }^{\circ} \mathrm{C}$ to $125^{\circ} \mathrm{C}$, allowing them to be accurately used over a typical industrial application temperature range. Furthermore, for control of electromechanical drive trains with significant resonances, the bandwidth of the SAW transducer and signal processing unit is typically $>2 \mathrm{kHz}$. The commercial inline transducer produces an analog output of $0.25 \mathrm{~V} / \mathrm{N} \cdot \mathrm{m}$ from a combined external signal processing unit and low-voltage power supply $(12 \mathrm{~V}, 1 \mathrm{~A})$. Nevertheless, the additional capital cost of the $20 \mathrm{~N} \cdot \mathrm{m}$ transducer, and associated electronics, is only a fraction of the cost of a similarly rated commercial servo machine and amplifier and is typically less than an integrated rotor position encoder.

\section{B. Experimental Results}

Experimental results now show the performance of the controllers in response to a 10-rad/s step reference demand, with a disturbance load torque of $2 \mathrm{~N} \cdot \mathrm{m}$ applied after $0.8 \mathrm{~s}$, thereby depicting the same events used for the simulation studies of Figs. 5, -7 , and 10. Fig. 12 shows responses from use of the PI controller of Fig. 2(a), where $K_{d}=0$. The PI gains are optimally tuned for the second-order model, i.e., for (8) and (11), for two values of $\omega_{x_{-} \text {pu }}$, with $R=1$ in both cases, thereby reflecting the simulation results described in Fig. 5. By comparison with Fig. 5, therefore, a good agreement between the tracking performance can be seen, with the regulation in response to the disturbance showing similar transient characteristics by virtue of having the same characteristic equation as for tracking [see (6) and (7), and (13) and (14)]. Furthermore, Fig. 12(b) shows results for the PI controller optimally tuned using the fourth-order model, i.e., from (7) and (12), for optimal $\omega_{x \_ \text {pu }}=0.88$ and $R=1$ and nonoptimal $R=2$ (i.e., the motor inertia is effectively halved by the removal of inertial disks), thereby reflecting the results of Fig. 6. It can be seen that while the gains chosen for optimal $R=1$ provide good responses for both the motor-side and load-side dynamics (cf. Fig. 6), the increase in inertia ratio to $R=2$ has only a minor influence on the induced dynamics (when $K_{p}$ and $K_{i}$ are recalculated assuming $J_{d}=J_{t} / 2$ ), thereby confirming the observations discussed in Section III-C, where it was stated that for $R>1$, the additional complexity afforded by the PID and $\mathrm{RRC}$ control structures is not necessary.

Fig. 13 shows measurements from the system used with the PID and RRC controllers and with $R=0.5$ (i.e., below the optimum value). The effective $K_{p}$ and $K_{i}$ gains are tuned to provide the same performance as those for results depicted

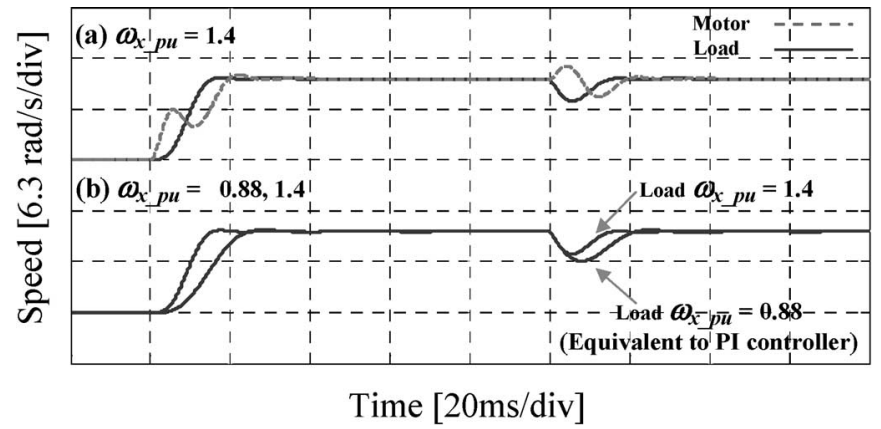

Fig. 10. Simulated tracking and regulation step responses of the RRC+ controller, where $R=1$.

TABLE IV

Optimal Tracking Performance Criteria and Regulation PERFORMANCE COMPARISON

\begin{tabular}{|c|c|c|c|}
\hline & \multicolumn{2}{|c|}{$\begin{array}{l}\text { Optimal Tracking } \\
\text { performance criteria }\end{array}$} & \multirow[t]{2}{*}{$\begin{array}{l}\text { Regulation performance comparison (Tracking } \\
\text { performance set optimal) }\end{array}$} \\
\hline & $\boldsymbol{R}$ & $\omega_{x p u}$ & \\
\hline$\overline{\text { PI }}$ & $\approx 1$ & 0.88 & $R \geq 1$, good performance. \\
\hline $\mathrm{PID} / \mathrm{RRC}$ & any & 0.88 & Same performance as PI. \\
\hline $\mathrm{RRC}+$ & any & any & $\begin{array}{l}\text { For any } R \text { increased performance over PI, PID and } \\
\text { RRC controllers as the closed-loop bandwidth ratio } \\
\text { is increased } \omega_{x p u}>0.88 \text {. }\end{array}$ \\
\hline
\end{tabular}

Note 1. Good tracking performance is obtained using PI controller when $R \geq 1$.

Note 2. For good performance the closed-loop bandwidth ratio of the PI, PID and RRC controllers is limited to $\omega_{x, p u}<1$

in Fig. 12(b) [see (7), (9), (10), and (12)]. However, the additional complexity afforded by the derivative action of the PID controller, and the extra complexity of the RRC, allows the inertia to be "tuned" to be $\tilde{R}=1$ by adjusting $K_{d}$ or $K_{s}$. From Fig. 13, it can be seen that both controllers provide near identical characteristics and are consistent with those for the optimally tuned PI controller in Fig. 12(b) for $R=1$, thereby demonstrating the equivalence of (6) and (7) for the PI, PID, and RRC controllers, respectively, when $\tilde{R}=1$.

To show the benefits of RRC+, Fig. 14 shows measurements from the system when the RRC+ controller gains are selected to induce user-designed values of optimal bandwidth, i.e., $\omega_{x}$ pu $=1,1.2$, and 1.4 (cf. with the simulation results of Fig. 10), with the mechanical system arranged such that $R=1$. It can be seen that while increasing bandwidth reduces rise time, as expected, good load-side dynamics remain for all cases. However, while maintaining optimum load-side performance, the motor-side dynamics deteriorate as a result of countering the effects of shaft compliance. For completeness, the lower trace of Fig. 14 compares the load-side performance for the proposed RRC+ controller and the basic PI controller. Since $R=1$, both can be tuned to provide optimal performance according to the ITAE polynomial (12). However, the benefit of employing the RRC+ is to allow an increase in closed-loop bandwidth without degrading the transient characteristics.

\section{Impact of Sensor Noise}

For all experimental cases presented previously, the derivative action required by the PID velocity control loop structure 


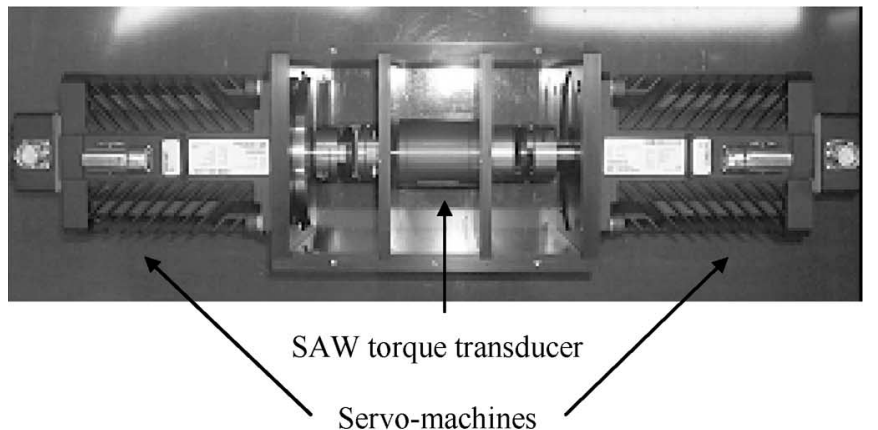

(a)

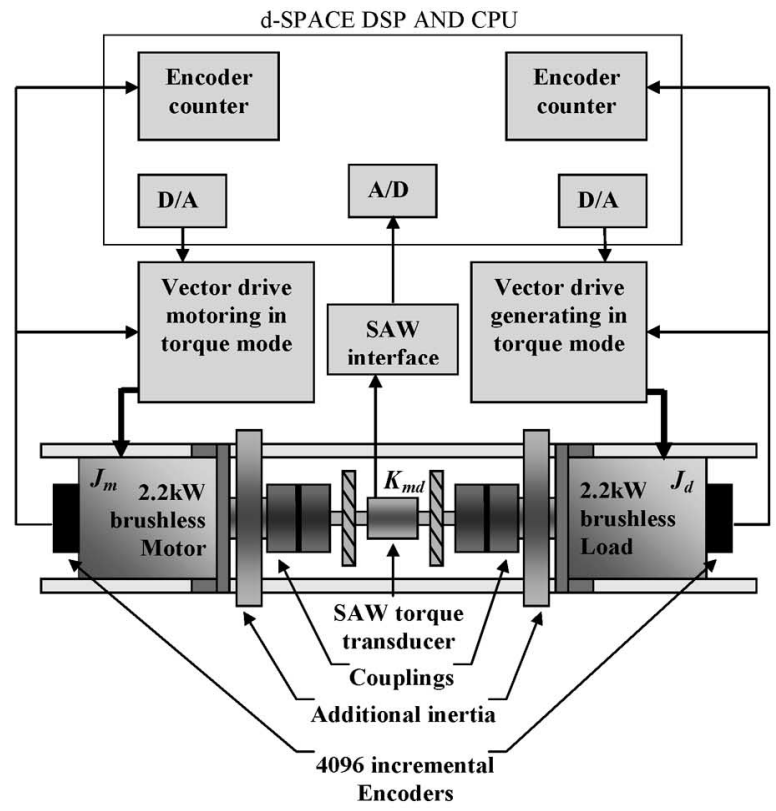

(b)

To RF couplers

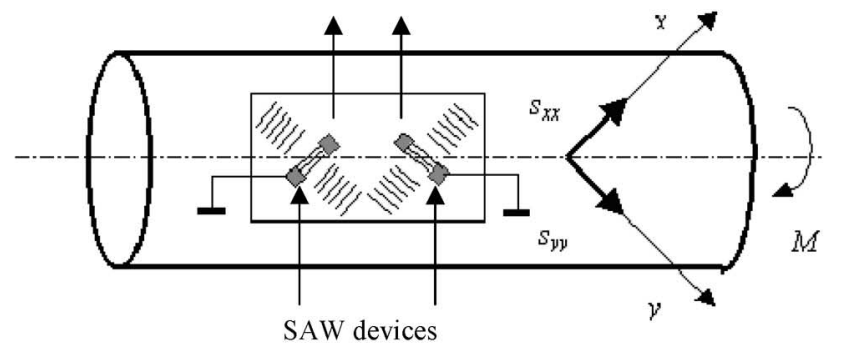

(c)

Fig. 11. Experimental facility, components, and control system. (a) Mechanical system overview. (b) Functions of components. (c) Torque-sensing elements based on SAW resonators.

is obtained from the double derivative of sensed motor shaft position via the provision of a 12-bit incremental encoder employing a quadrature position counter, i.e., motor acceleration $=$ $\left(d^{2} / d t^{2}\right) \theta_{m}$, where $\theta_{m}$ is the motor position. Due to the quantized nature of the feedback signal, noise is naturally injected into the closed-loop system and subsequently amplified by the derivative action of the PID. Since the required amount of derivative action increases as $R$ decreases (i.e., the amount of virtual inertia ratio increases (see Table II), where $K_{d}=J_{d}-$ $J_{m}$ ), noise levels induced by the feedback sensor have greater

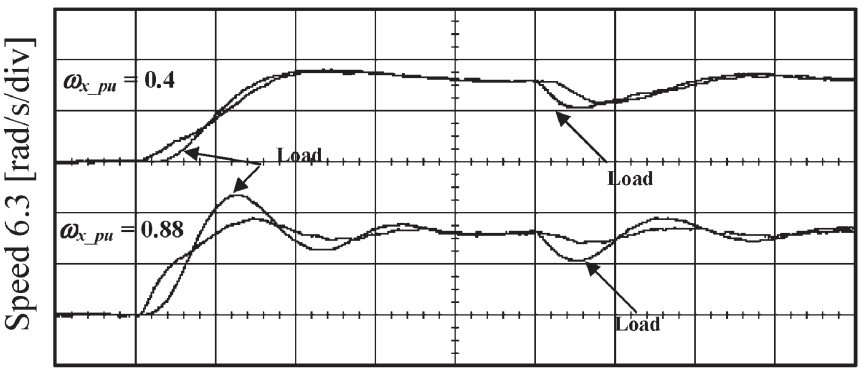

Time $[20 \mathrm{~ms} / \mathrm{div}]$

(a)

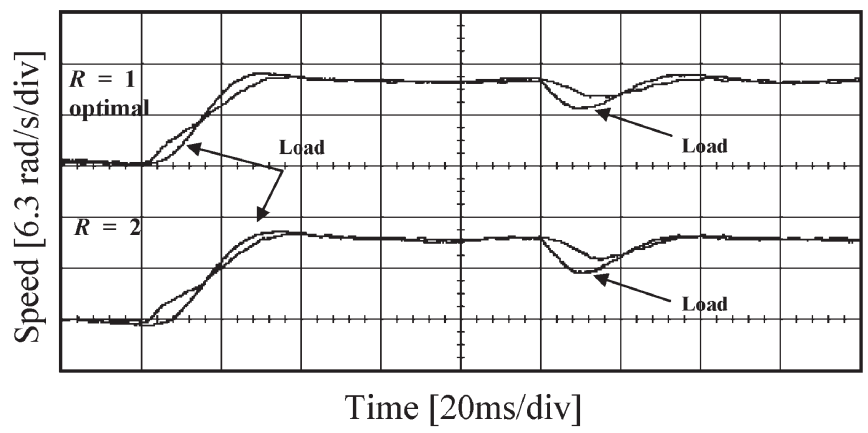

(b)

Fig. 12. Experimental tracking and regulation step responses of the PI controller. (a) Tuned to a single-inertia second-order model, where $R=1$. (b) Tuned to a two-inertia fourth-order model, where $R=1$.

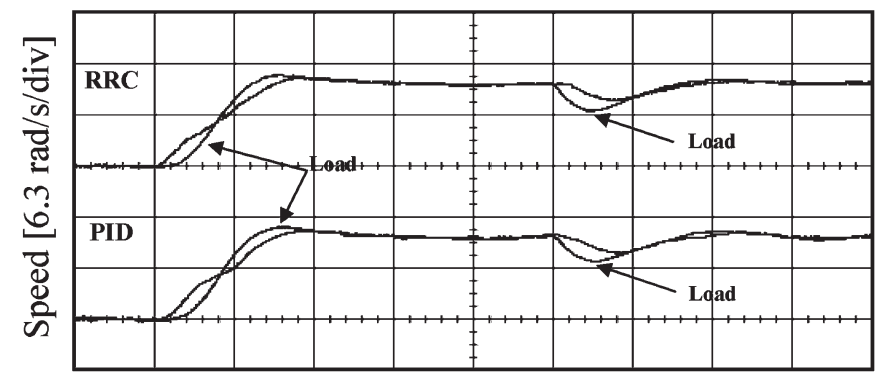

Time $[20 \mathrm{~ms} / \mathrm{div}]$

Fig. 13. Experimental tracking and regulation step responses for the RRC and PID controllers, where $R=0.5$ and $\tilde{R}=1$.

impact and ultimately lead to deterioration of the transient dynamics. For example, $J_{d}$ is now halved from $2.70 \times 10^{-3}$ to $1.35 \times 10^{-3} \mathrm{~kg} \cdot \mathrm{m}^{2}$ by the removal of inertial disks, thereby resulting in an increase in $\omega_{a}$ from 215.16 to $304.29 \mathrm{rad} / \mathrm{s}$, providing a mechanical inertia ratio of $R=0.25$. The modified mechanical parameters are therefore given in Table V.

Both the PID and RRC (which does not require the derivative action since it employs torque feedback) controllers are subsequently tuned according to the equations given in Table II, to provide optimal performance, i.e., $\tilde{R}=1, \omega_{x \_ \text {pu }}=0.88$. The resulting tracking and regulation transient responses for each scheme are shown in Fig. 15. It can be seen that while the RRC provides expected transient dynamics, the low-pass filter required to reduce noise in the system when employing the PID structure imparts a significant deterioration in performance by virtue of reducing stability margins. 


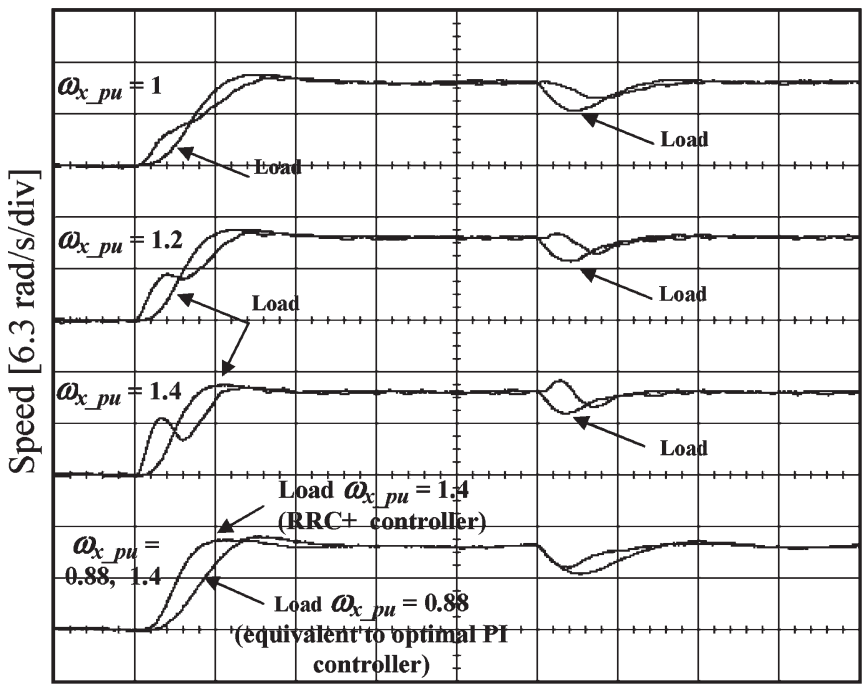

[Time $20 \mathrm{~ms} / \mathrm{div}]$

Fig. 14. Experimental tracking and regulation step responses for the RRC+ controller, where $R=1$.

It is notable that controllers that rely on estimates of shaft torque [4]-[6] via motor-side position sensors, as based on the relationship

$$
t_{\mathrm{md}}=t_{e}-J_{m} \frac{d^{2}}{d t^{2}} \theta_{m}
$$

also require a contribution from the double derivative of rotor position and are therefore essentially subjected to similar noise effects and, ultimately, to the same limitations as the classical PID structure.

Moreover, Sugiura and Hori [12] proposed a controller employing a signal proportional to the first derivative of the estimated torque using (18). Such a signal requires the triple derivative of encoder position, and the elevated noise levels may only be suppressed by low-pass filtering (over and above that required of a PID control scheme), thereby rendering it unsuitable for use in a high-bandwidth state-variable controller of the type considered herein, i.e., the proposed RRC+ controller. Nevertheless, since the RRC+ controller generally requires a derivative contribution of shaft torque and higher gains than its RRC counterpart, the closed-loop bandwidth is ultimately restricted in practice due to elevated noise levels. In an attempt to promote increased bandwidth, RRC+ was implemented via a signal proportional the difference in sensed motor and load speeds (using the first derivative of motor and load position; cf. (15)]; however, in so doing, no bandwidth advantage could be obtained. This was primarily a result of the relatively good signal-to-noise characteristics exhibited by the torque transducer measured at $\approx 55 \mathrm{~dB}$. Furthermore, the advantage of the proposed RRC+ scheme for the two-inertia system is that only variables sensed at the motor side, as opposed to the load side, are required.

\section{CONCLUSION}

Various compensation schemes have been investigated for the coordinated motion control of two-inertia mechanical sys-
TABLE $\mathrm{V}$

Modified Mechanical Parameters

\begin{tabular}{l|l}
\hline$R$ & 0.25 \\
$J_{d}$ & $0.00135 \mathrm{kgm}^{2}$ \\
$K_{m d}$ & $125 \mathrm{Nm} / \mathrm{rad}$ \\
$\omega_{a}$ & $304.29 \mathrm{rad} / \mathrm{s} \quad(48 H z)$ \\
\hline
\end{tabular}

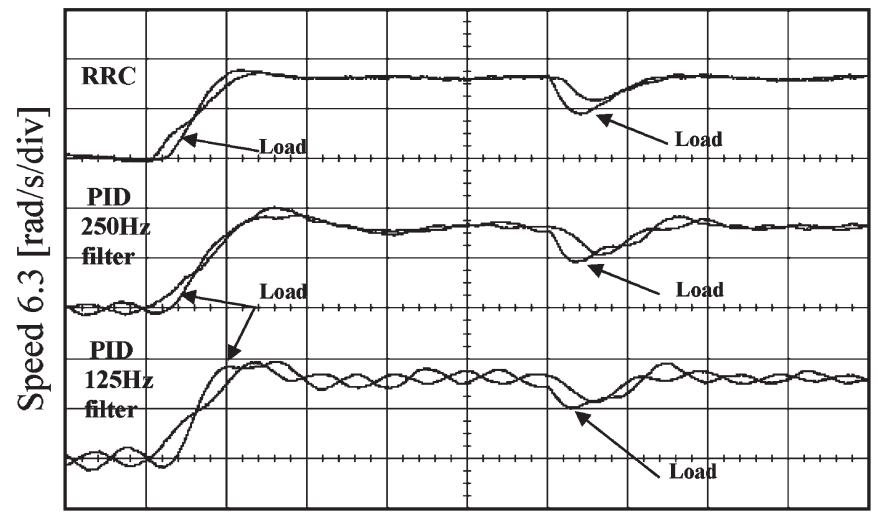

Time $[20 \mathrm{~ms} / \mathrm{div}]$

Fig. 15. Experimental tracking and regulation step responses for the RRC and PID controllers, where $R=0.25$ and $\tilde{R}=1$.

tems. The distinction between the resulting motor-side and load-side dynamics has been discussed, and the conditions that have been derived show when a dual-inertia system can be adequately described by a second-order closed-loop approximation and when an undesirable load-side performance will result, thereby necessitating the use of a fourth-order drivetrain model.

It has been shown that a basic second-order PI control scheme is adequate to provide good closed-loop performance when the inertia ratio of the motor and load is greater, or equal to unity, and the induced closed-loop bandwidth is less than $0.4 \times$ antiresonant frequency of the two-inertia system. Under converse conditions, the fourth-order model is shown to be necessary, and, while the PI control scheme can still impart optimal closed-loop characteristics (based on the ITAE criterion), no freedom exists for the designer to choose a desired closed-loop bandwidth and inertia ratio. Consequently, the extra flexibility afforded by the PID and RRC control schemes is shown to be sufficient to simultaneously impart optimal closedloop performance while also allowing the independent selection of virtual inertia ratio.

On a practical issue, although the PID and RRC controllers are demonstrated to be theoretically able to impart identical closed-loop dynamic attributes to the system, a qualitative study of the practical effects of using a double derivative of motor shaft position to generate the D of the PID controller, and the torque signal from a high-performance (albeit lowcost) torque sensor (a SAW-based device in this case), has shown that the RRC-type structure is a preferred solution. Moreover, the proposed RRC+ scheme is subsequently shown to additionally facilitate independent assignment of closed-loop bandwidth over and above that previously reported for the RRC control schemes without inducing problems associated 
with the requirement for a load-side position sensor or a signal proportional to the triple derivative of motor-side position.

\section{ACKNOWLEDGMENT}

The authors would like to thank the U.K. Engineering and Physical Science Research Council (EPSRC) and Sensor Technology, Ltd., Banbury, U.K., for the provision of an EPSRC CASE studentship.

\section{REFERENCES}

[1] S. N. Vukosavic and M. R. Stojic, "Suppression of torsional oscillations in a high performance speed servo drive," IEEE Trans. Ind. Electron., vol. 45, no. 1, pp. 108-117, Feb. 1998.

[2] G. Zhang and J. Furusho, "Speed control of a two-inertia system by PI/PID control," IEEE Trans. Ind. Electron., vol. 47, no. 3, pp. 603-609, Jun. 2000.

[3] G. Brandenburg and U. Schafer, "Cascade-state control of an elastic three mass system for cold rolling main drives," in Proc. IFAC Motion Control, Grenoble, France, 1998, pp. 139-151.

[4] Y. Hori, H. Sawada, and Y. Chun, "Slow resonance ratio control for vibration suppression and disturbance rejection in torsional system," IEEE Trans. Ind. Electron., vol. 46, no. 1, pp. 162-168, Feb. 1999.

[5] K. Yuki, T. Murakami, and K. Ohnishi, "Vibration control of 2-mass resonant system by resonance ratio control," in Proc. IEEE IECON, 1993, vol. 3, pp. 2009-2014.

[6] S. Mortimoto, A. Hamamoto, and Y. Takeda, "Vibration control of twomass system with low inertia ratio considering practical use," Electr. Eng. Jpn., vol. 125, no. 2, pp. 1-9, 1998.

[7] A. Pohl and F. Seifert, "Wirelessly interrogable surface acoustic wave sensors for vehicular applications," IEEE Trans. Instrum. Meas., vol. 46, no. 2, pp. 1031-1038, Aug. 1997.

[8] A. Lonsdale, "Dynamic rotary torque measurement using surface acoustic waves," Sensors, vol. 18, no. 10, pp. 51-56, Oct. 2001.

[9] T. M. O'Sullivan, N. Schofield, and C. M. Bingham, "Simulation and experimental validation of induction machines dynamics driving multiinertia loads," in Proc. 11th Int. Symp. Appl. Electromagn. and Mech. (ISEM), Versailles, France, 2003, Paper 01-4.

[10] _ "Simulation and experimental validation of induction machines dynamics driving multi-inertia loads," J. Appl. Electromagn. Mech., vol. 19, no. 1-4, pp. 231-236, 2003.

[11] R. C. Dorf, Modern Control Systems. Reading, MA: Addison-Wesley, 2000.

[12] K. Sugiura and Y. Hori, "Vibration suppression in 2-and 3-mass system based on the feedback of imperfect derivative of the estimated torsional torque," IEEE Trans. Ind. Appl., vol. 43, no. 1, pp. 56-64, Jan./Feb. 1996.

[13] Y. Hori, H. Iseki, and K. Sugiura, "Basic consideration of vibration suppression and disturbance rejection control of multi-inertia system using SFLAC (state feedback and load acceleration control)," IEEE Trans. Ind. Appl., vol. 30, no. 4, pp. 889-896, Jul./Aug. 1994.

[14] The Control Techniques Drives and Controls Handbook (IEE Power and Energy Series, 35), B. Drury, Ed. London, U.K.: Inst. Elect. Eng., 2001.

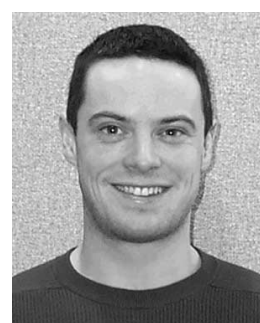

Tim M. O'Sullivan received the M.Eng. degree from the Department of Electronic and Electrical Engineering, University of Sheffield, Sheffield, U.K., in 2000 , where he is currently working toward the $\mathrm{Ph} . \mathrm{D}$. degree.

He has subsequently been investigating the use of surface acoustic wave torque transducers for improving the dynamic response of industrial servodrive systems with the Department of Electronic and Electrical Engineering, University of Sheffield.

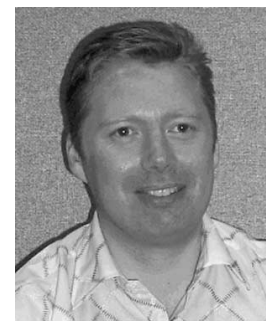

Chris M. Bingham (M'00) received the B.Eng. degree in electronic systems and control engineering from Sheffield City Polytechnic, Sheffield, U.K., in 1989, the M.Sc.(Eng.) degree in control systems engineering from the University of Sheffield, Sheffield, in 1990, and the Ph.D. degree for research on control systems to accommodate nonlinear dynamic effects in aerospace flight-surface actuators from Cranfield University, Cranfield, U.K., in 1994.

After earning his Ph.D. degree, he remained with Cranfield University as a Postdoctoral Researcher until subsequently taking up a research position with the University of Sheffield. Since 1998, he has been a Lecturer with the Department of Electronic and Electrical Engineering, University of Sheffield. His current research interests include traction control/antilock braking systems for electric vehicles, electromechanical actuation of flight control surfaces, control of active magnetic bearings for high-speed machines, sensorless control of brushless machines, and analysis and design of resonant converter systems.

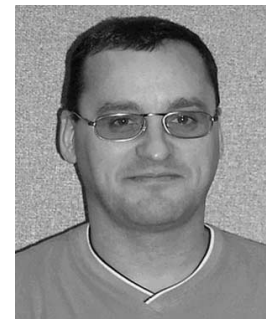

Nigel Schofield received the B.Eng. degree in electrical power engineering and the Ph.D. degree for research on the field weakening of brushless permanent-magnet traction machines from the University of Sheffield, Sheffield, U.K., in 1990 and 1997, respectively.

During the period 1993-1995, he served as a Senior Experimental Officer with the Department of Electronic and Electrical Engineering (EEE), University of Sheffield, before taking up the post of Design Engineer in industry. From 1997 to 2000, he was a Postdoctoral Researcher with the Electrical Machines and Drives Research Group, Department of EEE, University of Sheffield, and from 2000 to 2004, he was a Lecturer in this department. On July 1, 2004, he was appointed to a Mechatronics Lectureship with the School of Electrical and Electronic Engineering, University of Manchester, Manchester, U.K. His research interests include electromagnetic power trains for all-electric and hybrid-electric vehicles, the vehicular application of hydrogen fuel-cell systems, aerospace machines and actuators, and industrial applications of electromagnetic devices. 